\title{
GreenVLAN: An Energy-Efficient Approach for VLAN Design
}

\author{
${ }^{\dagger}$ Keqiang He, ${ }^{\dagger}$ Yi Wang, ${ }^{\ddagger}$ Xiaofei Wang, ${ }^{\dagger}$ Wei Meng, ${ }^{\dagger}$ Bin Liu \\ ${ }^{\dagger}$ Tsinghua National Laboratory for Information Science and Technology \\ ${ }^{\dagger}$ Department of Computer Science and Technology, Tsinghua University, China \\ ${ }^{\ddagger}$ School of Electronic Engineering, Dublin City University, Ireland
}

\begin{abstract}
The greening of the Internet has become an important research issue due to the huge energy consumption of the Internet. In this paper, we explore network-level power saving mechanisms in the context of virtual local area networks (VLANs) given their importance and prevalence in enterprise, campus and Data Center Networks (DCNs). After investigating the disadvantages of current VLAN design method, we propose the GreenVLAN approach, which aims at reducing the power usage in the next generation Energy-Efficient Ethernet. The proposed GreenVLAN approach is formulated as a nonlinear integer programming problem with the total energy consumption as the objective to be minimized. Besides, we develop a practical heuristic algorithm to solve the proposed model and the complexity of the algorithm is analyzed. Our experiments on both simulated topology and real-world campus network demonstrate that GreenVLAN could achieve considerable power saving (reduce the power consumption of Ethernet links by about $27 \% \sim 53 \%$ for a typical VLAN configuration) compared with current VLAN practice.
\end{abstract}

\section{INTRODUCTION}

Currently, reducing the huge energy consumption of the Internet has become an important issue for both research and industry communities. The energy usage of the network infrastructure in the United States has been estimated to be at $5 \sim 24 \mathrm{TWh} /$ year [1]. With the increasing development of cloud computing and data centers, the situation is getting worse. LAN switches and routers comprise the bulk of network devices and they consume the largest percentage of network energy usage (more than $50 \%$ of the total energy consumption according to [2]). Thus, the IEEE has been developing Energy-Efficient Ethernet (EEE) [3] to make Ethernet connections more power efficient. It is pointed out that the power saving technology of the Ethernet interface cards in US alone could save companies between $\$ 300$ million and $\$ 450$ million per year [4].

Virtual Local Area Networks (VLANs) are extensively used in Ethernet networks. They are widely deployed to reduce management complexity, to improve network performance and security in enterprise, campus and Data Center Networks (DCNs), etc [5] [6] [7] [8]. Traditionally, VLANs are comprised of users belonging to the same category, permitting hosts connected to LAN switches or routers to be grouped together into logical groups despite of their physical location. Typically, VLANs are created and removed by network operators. Hosts in the same logical group (for example, faculties or employees in the same department) are usually classified into a number of VLANs. Traffic loads are spread via a Spanning-Tree [9] [10], which spans all the switches or routers in LAN and provides path redundancy while eliminating undesirable loops in the network. Traffic between different VLANs is routed through designated routers.

There are a number of literatures investigating power management schemes to reduce energy consumption of networks. Recent power management approaches have been mainly focused on the PCs, labtops, and network equipments such as routers, switches, Network Interface Controllers (NICs) and other devices [11][12][13][14][15][16]. However, few power management schemes have been proposed to achieve power saving from the network level, namely, at the Layer 2 or Layer 3 level. Sergiu Nedevschi et al. present two forms of schemes [1][12]. The first is based on putting network elements into sleep stand mode during idle periods, the other is that network devices should adapt to the traffic load on the link, dynamically setting the packet processing rate. Maruti Gupta et al. [14] investigate the possibility of putting various network components on LAN switches into sleep during periods of low traffic activity. Their research results indicate the feasibility of power savings on LAN switches and routers using sleeping and low power mode. Different from the existing approaches that treat switches or routers as isolated devices, we concentrate on developing mechanisms that use EEE more effectively to design energy-efficient VLANs because of their importance and prevalence.

In this paper, we propose GreenVLAN, an energy-efficient approach for VLAN design. Broadcast traffic costs, the actual flow path versus shortest physical path and the traffic matrix among hosts are taken into account in designing GreenVLAN. Specifically, our main contributions are:

1) We propose the GreenVLAN solution to reduce the power consumption. A nonlinear integer programming model is developed to minimize the total power usage in the network. Besides, we present a practical heuristic algorithm to solve the proposed model and analyze the time complexity of the algorithm.

2) We evaluate the potential power savings using both simulated network environment and real-world topology. Our experimental results demonstrate that, for a typical VLAN configuration, the GreenVLAN would reduce the energy consumption of Ethernet links by about $27 \% \sim 53 \%$, compared with the traditional approach.

The rest of the paper is organized as follows. Section II 
reviews the previous work. Section III presents VLAN basics, our assumptions and the problem statement. In Section IV, the GreenVLAN model is developed and a heuristic algorithm is given. Section V evaluates GreenVLAN using both simulated and real-world topologies. Section VI concludes the paper.

\section{RELATED WORK}

Maruti Gupta et al. in [2] address the problem of energy consumption of the overall network, which might be the pioneer in this area. They use data from the US Department of Commerce to demonstrate the huge energy consumption of the Internet and point out that there are no Energy Star recommendations for networking equipment. Using sample packet traces, they reveal that it is indeed reasonable to put network interfaces and other network device components to sleep. Besides they discuss the impacts on network protocols by putting network interfaces and other router or switch components to sleep.

At the network level, Zhang et al. propose an power-aware traffic engineering mechanism in [17]. They notice the fact that today's network has high path redundancy and low link utilization. Their basic idea is to build models to maximize the number of links that can be put into sleep while honoring some practical constraints such as link utilization and packet dealy. The evaluation results on real-world network topology and traffic data reveal that the power-aware traffic engineering proposal can reduce line-cards' power consumption by $27 \%$ to $42 \%$.

In the context of VLAN design, the authors of [5] investigate the VLAN usage in enterprise networks. They find that despite of their extensive prevalence in enterprise and campus networks, current VLANs are usually inefficiency due to the suboptimal placement policies. While VLAN simplifies management, it may introduce longer paths for data flows and longer delay. So they argue that minimizing inefficiencies requires operators to carefully place designated routers. Besides, they find that the process of configuring VLANs is error-prone and complex. These error-configured VLANs lead to hosts being disconnected, and result in unnecessary broadcast traffic. Xin Sun et al. [6] find that VLAN configuration is ad-hoc and errorprone. Frequent changes are always needed in VLAN design. So they present algorithms to decide the process of VLAN partition automatically.

\section{BACKGRound AND PRoblem Statement}

\section{A. VLAN Basics}

As illustrated in Figure 1, there are three VLANs in the network. Hosts $\mathrm{C} 1, \mathrm{C} 2, \mathrm{C} 5$ and $\mathrm{C} 8$ belong to VLAN 1. Similarly, hosts C3, C4, C6 and C9 belong to VLAN 2. Hosts $\mathrm{C} 7$ and $\mathrm{C} 8$ belong to VLAN 3. Assume that hosts in VLAN 1 and VLAN 2 belong to a category and hosts in VLAN 3 belong to another one. S1-S6 are switches. R1, R2 are routers. Traffic within a certain VLAN is spread using a Spanning Tree. For example, the bold lines illustrate the Spanning Tree of VLAN 1. The traffic from $\mathrm{C} 1$ to $\mathrm{C} 5$ is forwarded along $\mathrm{C} 1 \rightarrow \mathrm{S} 1 \rightarrow \mathrm{S} 2 \rightarrow \mathrm{S} 3 \rightarrow \mathrm{C} 5$. Each VLAN is associated with a designated router, for example, in Figure 1, router R1 and R2 are the

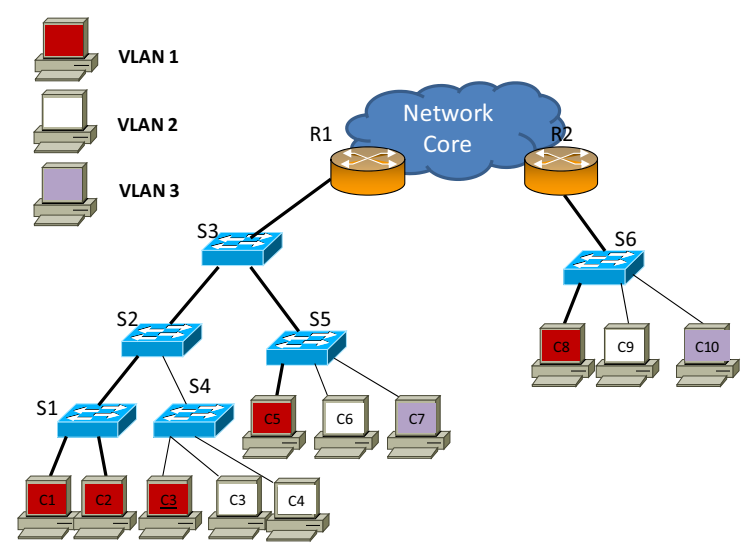

Fig. 1. An illustration of energy-efficient VLAN design.

designated router for VLAN 1 and VLAN 2 respectively. When hosts in a VLAN communicate with hosts outside the VLAN, the traffic would be routed through the designated router. For example, in Figure 1, the data path between host $\mathrm{C} 1$ and host $\mathrm{C} 6$ is $\mathrm{C} 1 \rightarrow \cdots \rightarrow \mathrm{R} 1 \rightarrow \cdots \rightarrow \mathrm{R} 2 \rightarrow \cdots \rightarrow \mathrm{R} 1 \rightarrow \cdots \rightarrow \mathrm{C} 6$.

\section{B. Assumptions and Problem Statement}

Before presenting our GreenVLAN approach, we make the following assumptions:

- Network equipments such as routers, switches and NICs support energy-efficient schemes, in other words, they could be put into low power mode (sleeping or low operating rate) according to the traffic volume exchanged on the links.

- The traffic behavior appears recently could represent the traffic behavior in the near future.

Building green Internet requires not only energy efficiency schemes for network devices but also power-aware network management mechanisms. The VLAN partition procedure must obey correctness criteria and feasibility criteria first (see section IV-A). Nevertheless, current VLAN practice is energy inefficiency. There are three main disadvantages in current VLAN design. Firstly, some VLANs created by network operators have too many hosts and maintain quite large Spanning Trees. In this case, the broadcast traffic spread in the network would be quite significant. Then, the opportunity that switches and routers running at low power mode is small. Consequentially, the power saving opportunity is reduced. Secondly, the traditional VLAN partition solution might be non-optimization from the standpoint of network performance and energy efficiency. The actual data flow path between two hosts in a VLAN is usually much longer than the shortest physical path between the two hosts according to [5]. Hence, traffic would be switches on more links and more power would be consumed by network devices. Thirdly, current VLANs are created without taking traffic pattern into consideration. Nevertheless, traffic among hosts may vary during the time (such as large storage file sharing in Storage Area Network). For example, host C3 may be divided into VLAN 2 according to the network operator. However, it is probably that at host C3 exchanges large 
volume of data with the hosts in VLAN 1 (for instance, large storage file sharing) while little traffic is exchanged with other hosts. According to the original VLAN design, more traffic would be switched or routed on the links. In Figure 1 , data flows along $\mathrm{C} 1 \rightarrow \mathrm{S} 1 \rightarrow \mathrm{S} 2 \rightarrow \mathrm{S} 3 \rightarrow \mathrm{R} 1 \rightarrow \cdots \rightarrow \mathrm{R} 2 \rightarrow \cdots \rightarrow$ $\mathrm{R} 1 \rightarrow \mathrm{S} 3 \rightarrow \mathrm{S} 2 \rightarrow \mathrm{S} 4 \rightarrow \mathrm{C} 3$. If host $\mathrm{C} 3$ is grouped into VLAN 1, then the traffic would be exchanged through only 4 links, that is $\mathrm{C} 1 \rightarrow \mathrm{S} 1 \rightarrow \mathrm{S} 4 \rightarrow \mathrm{C} 3$. When the traffic volume between host $\mathrm{C} 1$ and C3 is large, the total traffic volume reduced will be quite significant. Therefore, to reduce the energy usage in EEE, we develop network level power saving mechanisms to overcome the problems stated above and help network operator design energy-efficient VLANs.

\section{GREENVLAN}

In this section, we develop our models and algorithm to design energy-efficient VLANs. For convenience, we summarize the main notations used of this paper in TABLE I.

\section{A. GreenVLAN Model}

Generally speaking, VLANs should be created according to several criterion [6]. These practical criterion constrain the partition procedure. These criterion are:

- correctness criteria: Hosts in different categories must be grouped into different VLANs for security and privacy reasons.

- feasibility criteria: In practical VLAN design, the number of hosts per VLAN is restricted by the size of the network IP block, the maximum number of VLANs allowed is determined by the hardware constrain, i.e., the maximum number of STPs switches can support .

- performance and cost criteria: Keeping the broadcast traffic of each VLAN small is quite important. Typically, the broadcast traffic is determined by three factors: the number of hosts in the VLAN, the average broadcast traffic of each host and the span of the VLAN (the number of links in the spanning tree). Here we employ the broadcast traffic model used in [6]. The broadcast traffic could be denoted as $B=M * A * W$, where $M$ represents the number of hosts, $A$ denotes the average broadcast traffic of hosts and $W$ means the number of links in the spanning tree.

Let $T_{i j}$ denote the traffic volume from host $i$ to host $j$ $\left(T_{i j}=0\right.$ if $\left.i=j\right) . x_{i j}$ denotes whether host $i$ and host $j$ belong to the same VLAN $\left(x_{i j}=0\right.$ if $\left.i=j\right)$. $F_{l}$ denotes the traffic demand of link $l$. The energy consumption of link $l, E_{l}$ depends on $F_{l}$. Here we assume $E_{l}=\Psi\left(F_{l}\right)$, where $\Psi$ is energy function. The objective of the GreenVLAN model is to minimize the total energy consumption of the network while guaranteing the three kinds of criteria described above. Specifically, the proposed GreenVLAN model is described by
TABLE I

TABLE OF NOTATIONS.
Equation 1 to Equation 7.

$$
\begin{array}{ll}
\min & \sum_{l \in L} E_{l} \\
\text { s.t. } & E_{l}=\Psi_{l}\left(F_{l}\right) \\
& \sum_{j=1}^{h} x_{i j} \leq N, \forall i \in H \\
& \sum_{j=1}^{h}\left(1-x_{i j}\right) \leq M, \forall i \in H \\
& x_{i j}(1-Q(i, j))=0, \forall i, j \in H \\
& x_{i j} x_{j k}-x_{i k}=0, \forall i, j, k(i<j<k) \in H \\
& B(v(i))=\sum_{j=1}^{h} x_{i j} * A(v(i)) * W(v(i)) \leq T h, \forall i \in H
\end{array}
$$

Equation 1 computes the total energy consumption in the network. Equation 2 calculates the energy usage of link $l$. Equation 3 and Equation 4 guarantee that the produced grouping solution should satisfy the feasibility criteria, that is, the number of host per VLAN should not exceed the upper bound $N$ and the number of VLANs created should not exceed the upper bound $M$. Equation 5 means the produced grouping solution should guarantee the correctness criteria, in other words, hosts grouped into the same VLAN must belong to the same logical category. Equation 6 means that if $x_{i j}=1$ and $x_{j k}=1$, then $x_{i k}$ must also equals to $1(i<j<k)$, this equation guarantees the correctness of the solution. Equation 7 makes sure that for each created VLAN, the broadcast traffic will not overflow the threshold $T h$, which might be determined by the network operator.

In EEE, the energy function $\Psi$ is a real increasing function according to [11]. In other words, the energy consumption grows when the traffic load increases. Minimizing the total traffic volume exchanged in the overall network could maximize 
the total power saving. Thus, the above model (Equation 1 and Equation 2) can be refined as:

$$
\begin{array}{ll}
\min & \sum_{\forall i, j \in H} p_{i j} T_{i j} \\
\text { s.t. } & p_{i j}= \\
& \left\{\begin{array}{rl}
d_{i j}, & x_{i j}=1 \\
d_{i r_{1}}+d_{j r_{2}}+d_{r_{1} r_{2}}, & x_{i j}=0
\end{array}, \quad i, j \in H, i \neq j\right.
\end{array}
$$

Here, the data path length $p_{i j}$ means the number of links a packet passed from its source host to the destination. When host $i$ and host $j$ are grouped in the same VLAN, the data path from host $i$ to host $j$ equals to $d_{i j}$, where $d_{i j}$ is the shortest path length from host $i$ to host $j$. When the two hosts are not in the same VLAN, for example, host $i \in$ VLAN 1 and host $j \in \mathrm{VLAN} 2$, then $p_{i j}$ can be represented by $d_{i r_{1}}+d_{j r_{2}}+d_{r_{1} r_{2}}$, where $r_{1}$ and $r_{2}$ denote the designated router for VLAN 1 and VLAN 2 respectively.

The objective of the GreenVLAN model is to find a solution for the binary (integer) variable $x_{i j}(i, j=1,2 \cdots h, i<j)$ which minimizes $\sum p_{i j} T_{i j}$. In fact, the proposed model is a nonlinear integer programming problem with constraints. Generally, this kind of problems is NP-hard [18]. Thus, we develop a practical algorithm to solve this model which will be introduced in the following subsection.

\section{B. A Practical Algorithm}

In order to solve the nonlinear integer programming model proposed above, we develop a practical algorithm to find a reasonable VLAN partition that converges to optimal solution. The algorithm has the following steps:

Step1: Assume there are $K$ logical categories in the network, then at least $K$ VLANs must be created according to the correctness criteria. Suppose the hosts in VLAN $k(k=1,2 \cdots K)$ is $H_{k} . T=\left\{T_{i j}\right\}$ and $D=\left\{d_{i j}\right\}$. If VLAN $k$ satisfies the constraints described by Equation 3 to Equation 7, then output VLAN $k$, else turn to Step 2.

Step2: Because VLAN $k$ does not satisfy the constraints, we need to divide it into several VLANs while converging to the most energy-efficient solution. Here we define a heuristic factor $\beta_{i j}$ :

$$
\beta_{i j}=T_{i j} / d_{i j}, i \neq j
$$

If host $i$ and host $j$ have large amount of traffic $T_{i j}$ and have small $d_{i j}$, then it is most likely the two hosts should be grouped into the same VLAN. Otherwise the amount of traffic $T_{i j}$ will be switched on more links which obviously aggravate the traffic volume of other links and hence the energy consumption. Thus, we employ $\beta_{i j}$ as the criteria and use k-means cluster algorithm to partition $H_{k}$.

\section{EVALUATION}

In this section, we evaluate the energy saving of the proposed GreenVLAN using simulated and real-world topologies. Besides, we compare our GreenVLAN with traditional VLAN practice.

\section{A. Experimental Setup}

We design a simulated topology here as shown in Figure 2. We assume the switches in this topology have 48 ports and there are 40 hosts connected to each switch. Six switches are connected as an UNIT, and six such UNITs are connected by a router to constitute the whole topology. So there are about 1,500 hosts in our simulation. For convenience, the cental router in Figure 2 is used as the designated routers for all created VLANs. We use the Layer 2 topology on the Georgia Tech network [8] as the real-world topology. In this topology, the switches at the periphery belong to various departments, each department is associated with a central switch which is connected to several core routers. Similarly, we assume each switch is associated with 40 hosts in our simulation.

We generate two kinds of traffic patterns. The first is that the traffic volume between any two host follows a exponentially distribution (the location parameter $\lambda=0.5$, i.e., the mean traffic volume is 2 (Mbps)). The second is that the traffic volume between any two host follows a truncated Pareto distribution (the shape parameter is 1.02 and the scale parameter is 3 and we only select values smaller than 5(Mbps)). We assume the broadcast traffic is $1 \mathrm{kbps}$ per host (approximately 2 packets/s per host according to [6]) and Th is set to $60 \mathrm{Mbps}$ for the simulated topology and $200 \mathrm{Mbps}$ for the GIT campus network.

In our evaluation, we mainly focus on the power usage of the ethernet links. Given the topology and traffic matrix, we can get an energy-aware VLAN partition solution and a traditional VLAN partition solution. Then, it is easy to get the data path of each pair of hosts . Consequently, the traffic volume on each links can be calculated. The energy consumption of each link is estimated according the the evaluation results of [11]. So the energy consumption of GreenVLAN and traditional VLAN could be calculated respectively.

\section{B. Experimental Results}

We denote the energy consumption of GreenVLANs as $E_{1}$, the energy consumption of Tradditional VLANs as $E_{2}$. We use the following equation to quantify the potential power saving of the proposed GreenVLAN approach:

$$
\gamma=\frac{E_{1}}{E_{2}}
$$

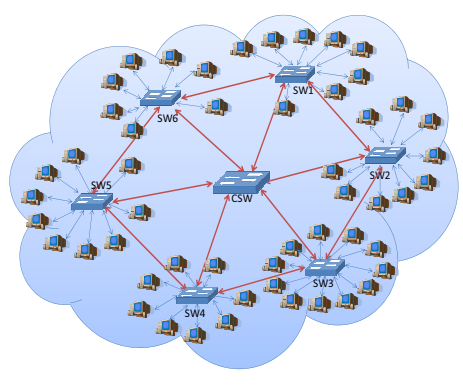

(a) An Unit

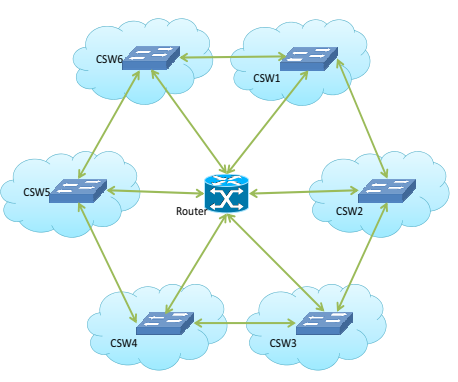

(b) Six Units Comprise of the Network

Fig. 2. The simulated topology. 


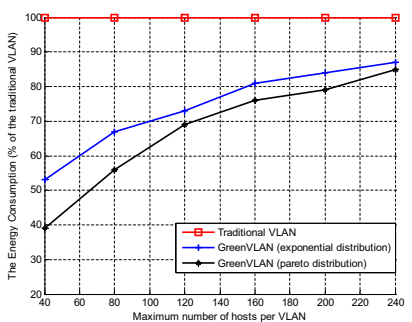

Fig. 3. Experiment results on simulated topology.

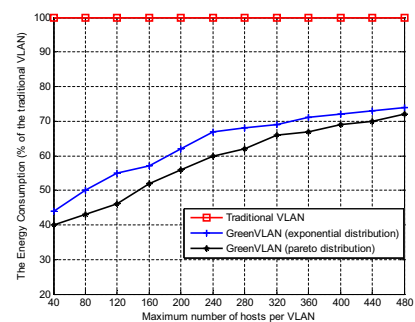

Fig. 4. Experiment results on GIT campus topology.
Here, traditional VLAN partition means that VLANs are created just according to the hosts' functionality and topology, i.e., the input of k-means algorithm is matrix $D$. Smaller $\gamma$ represents greater power saving of the GreenVLAN approach.

The experimental results on the simulated topology are shown in Figure 3. The x-axis represents $N$, i.e., the maximum number of hosts per VLAN. The y-axis represents $\gamma$. When the traffic among hosts is exponentially distributed, the proposed GreenVLAN consumes about 13\% 47\% less power than the traditional approach; when the traffic among hosts is pareto distributed, GreenVLAN reduces the total power consumption by about $15 \%$ to $51 \%$. According to the traditional approach, more traffic would be switched if $N$ is smaller. However, in GreenVLAN, hosts with large amount of traffic and short distance are grouped together. Therefore traffic switched is reduced, the total power usage would be reduced. When $N$ is large, the GreenVLAN converges to the traditional approach (the probability of hosts which communicate a lot to be grouped into a VLAN grows when $N \rightarrow h$ ), that is the reason why $\gamma$ grows with the increase of $N$.

Similarly, the experimental results on the GIT campus topology are shown in Figure 4. We can observe that GreenVLAN achieves about $27 \% \sim 56 \%$ and $29 \% \sim 59 \%$ power saving for exponentially distributed traffic pattern and pareto distributed traffic pattern respectively.

We have studied the potential power saving achieved by our GreenVLAN. According to [6], there are about 120 categories and 15000 hosts on in a large segment of the campus network. Many VLAN are small and a medium VLAN has about 80 hosts [6]. Large VLANs bring about large broadcast traffic and performance inefficiency (large packet delay and long flow path, etc). Based on the practical VLAN configuration case as well as theoretical consideration, we assume there are about 120 hosts in a VLAN, then our GreenVLAN would achieve about $27 \% \sim 53 \%$ power saving according to our simulation. VLANs are operated day and night, if the GreenVLAN approach is introduced in the next generation EEE, then significant power saving would be achieved.

\section{Conclusions}

Building green Internet requires not only energy-efficient network devices but also power-aware network design approaches. In this paper, we propose a novel energy-efficient VLAN design approach, GreenVLAN, which aims at minimizing the total power usage of network links in the next generation
Energy-Efficient Ethernet (EEE). In EEE, the objective of the proposed GreenVLAN model could be refined as minimizing the total traffic transferred in the network. So we refine our model and a nonlinear integer programming model is given. Then we present a practical heuristic algorithm to solve the proposed model. Experiments on both simulated topology and real-world topology demonstrate that considerable power saving could be achieved by the GreenVLAN approach compared with traditional VLAN practice.

\section{REFERENCES}

[1] C. Gunaratne, K. Christensen, B. Nordman, and S. Suen, "Reducing the energy consumption of Ethernet with adaptive link rate (ALR)." Published by the IEEE Computer Society, 2007, pp. 448-461.

[2] M. Gupta and S. Singh, "Greening of the Internet," in Proceedings of the 2003 conference on Applications, technologies, architectures, and protocols for computer communications. ACM, 2003, pp. 19-26.

[3] "IEEE 802.3 Energy Efficient Ethernet Study Group." [Online]. Available: http://grouper.ieee.org/groups/802/3/eeestudy/

[4] P. Reviriego, J. Hernadez, D. Larrabeiti, and J. Maestro, "Burst Transmission for Energy-Efficient Ethernet," Internet Computing, pp. 50-57, 2010.

[5] P. Garimella, Y. Sung, N. Zhang, and S. Rao, "Characterizing VLAN usage in an operational network," in Proceedings of the 2007 SIGCOMM workshop on Internet network management. ACM, 2007, pp. 305-306.

[6] X. Sun, Y. Sung, S. Krothapalli, and S. Rao, "A systematic approach for evolving VLAN designs," in Proceedings of 2010 INFOCOM. IEEE, 2010, pp. 1-9.

[7] Y. Sung, S. Rao, G. Xie, and D. Maltz, "Towards systematic design of enterprise networks," in Proceedings of the 2008 ACM CoNEXT Conference. ACM, 2008, pp. 1-12.

[8] M. Bin Tariq, A. Mansy, N. Feamster, and M. Ammar, "Characterizing VLAN-induced sharing in a campus network," in Proceedings of the 9th ACM SIGCOMM conference on Internet measurement conference. ACM, 2009, pp. 116-121.

[9] P. Medagliani, G. Ferrari, G. Germi, and F. Cappelletti, "Simulationassisted analysis and design of STP-based networks," in Proceedings of the 2nd International Conference on Simulation Tools and Techniques. ICST (Institute for Computer Sciences, Social-Informatics and Telecommunications Engineering), 2009, pp. 1-8.

[10] Cisco, "Understanding Spanning-Tree Protocol," 2005. [Online]. Available: http://dx.doi.org/10.1016/j.comnet.2005.01.011

[11] P. Reviriego, J. Hernández, D. Larrabeiti, and J. Maestro, "Performance evaluation of energy efficient ethernet," Communications Letters, IEEE, vol. 13, no. 9, pp. 697-699, 2009.

[12] S. Nedevschi, L. Popa, G. Iannaccone, S. Ratnasamy, and D. Wetherall, "Reducing network energy consumption via sleeping and rate-adaptation," in Proceedings of the 5th USENIX Symposium on Networked Systems Design and Implementation. USENIX Association, 2008, pp. 323-336.

[13] H. Jung, A. Hwang, and M. Pedram, "Predictive-flow-queue-based energy optimization for gigabit ethernet controllers," IEEE Transactions on Very Large Scale Integration (VLSI) Systems, vol. 17, no. 8, pp. 1113-1126, 2009.

[14] M. Gupta, S. Grover, and S. Singh, "A feasibility study for power management in LAN switches," in Proceedings of the 12th IEEE International Conference on Network Protocols (ICNP). Published by the IEEE Computer Society, 2004.

[15] C. Gunaratne, K. Christensen, and B. Nordman, "Managing energy consumption costs in desktop PCs and LAN switches with proxying, split TCP connections, and scaling of link speed," International Journal of Network Management, vol. 15, no. 5, pp. 297-310, 2005.

[16] M. Gupta and S. Singh, "Energy conservation with low power modes in Ethernet LAN environments," in IEEE INFOCOM Minisymposium, 2007.

[17] M. Zhang, C. Yi, B. Liu, and B. Zhang, "GreenTE: Power-Aware Traffic Engineering," in IEEE ICNP, 2010.

[18] M. Bazaraa, H. Sherali, and C. Shetty, Nonlinear programming: theory and algorithms. LibreDigital, 2006. 